\title{
Boundary-reaction-diffusion model for oscillatory zoning in binary crystals grown from solution
}

\author{
Felix Kalischewski, ${ }^{1,2}$, 田 Ihor Lubashevsky, ${ }^{1,3, \text { 由 }}$ and Andreas Heuer ${ }^{1,2, \text { I }}$ \\ ${ }^{1}$ Westfälische Wilhelms Universität Münster, Institut für physikalische Chemie, Corrensstr. 30, 48149 Münster, Germany \\ ${ }^{2}$ Center of Nonlinear Science CeNoS, Westfälische Wilhelms Universität Münster, 48149 Münster, Germany \\ ${ }^{3}$ A.M. Prokhorov General Physics Institute, Russian Academy of Sciences, Vavilov Str. 38, Moscow, 119991 Russia
}

(Dated: December 11, 2018)

\begin{abstract}
Oscillatory Zoning (OZ) is a phenomenon exhibited by many geologically formed crystals. It is characterized by quasi periodic oscillations in the composition of a solid solution, caused by selforganization. We present a new model for OZ. The growth mechanism applied includes species diffusion through the solution bulk, particle adsorption, surface diffusion and subsequently desorption or incorporation into the crystal. This mechanism, in particular, can provide the synchronization effects necessary to reproduce the layered structure of experimentally obtained crystals, lacking in other models. We conduct a linear stability analysis combined with numerical simulations. Our results reproduce the experimental findings with respect to the patterns formed and a critical supersaturation necessary for OZ to occur.
\end{abstract}

PACS numbers: 47.54.-r, 81.10.AJ, 05.65.+b, 82.40.ck

\section{INTRODUCTION}

Oscillatory zoning (OZ) is a phenomenon describing repetitive composition variations of binary solid solutions along their core-to-rim profile. Traditionally, it was believed to be of rare occurrence and its existence was ascribed to variations of external parameters controlling the crystal growth, like temperature or concentration fluctuations. However, the development of more sophisticated observation techniques facilitated the detection of this phenomenon in all major classes of minerals and a wide range of geological environments [1]. In addition to naturally obtained samples $\mathrm{OZ}$ was experimentally reproduced in the absence of external fluctuations. Reeder et al. 2] were able to grow calcite crystals exhibiting $\mathrm{OZ}$ of the $\mathrm{Mg}$ dopant and Putnis et al. [3, 4, 5] obtained end-member zoning in $(\mathrm{Ba}, \mathrm{Sr}) \mathrm{SO}_{4}$ crystals.

The experimental setup used by Putnis et al. [3, 4, 5, is sketched in Fig. 10 It consists of two reservoirs, one filled with an aqueous solution of $\mathrm{BaCl}_{2} / \mathrm{SrCl}_{2}$ and the other with $\mathrm{Na}_{2} \mathrm{SO}_{4}$. The two reservoirs are connected by a column filled with a silica-gel to inhibit convective transport. With the beginning of the experiment the reactants from the reservoirs start to diffuse toward each other through the column. As the diffusion fields of $\mathrm{Ba}^{2+}$ and $\mathrm{Sr}^{2+}$ from one reservoir and $\mathrm{SO}_{4}{ }^{2-}$ from the other reservoir exceed the nucleation threshold product in the vicinity of the column center, nuclei form. The solution is then strongly supersaturated with respect to the freshly generated crystal seeds and the growth commences in a layer of few millimeters in width $[\underline{6}$ ]. After approximately one month the experiment was terminated. The obtained

\footnotetext{
*Electronic address: Kalischewski@uni-muenster.de

${ }^{\dagger}$ Electronic address: ialub@fpl.gpi.ru

‡Electronic address: andheuer@uni-muenster.de
}

crystals exhibited OZ although no external fluctuations were imposed on the system. Thus it has been clearly shown that $\mathrm{OZ}$ can be also attributed to intrinsic mechanisms resulting in spontaneous structure formation []]. The wide range of different crystals concerned suggests a certain universality of the underlying mechanism.

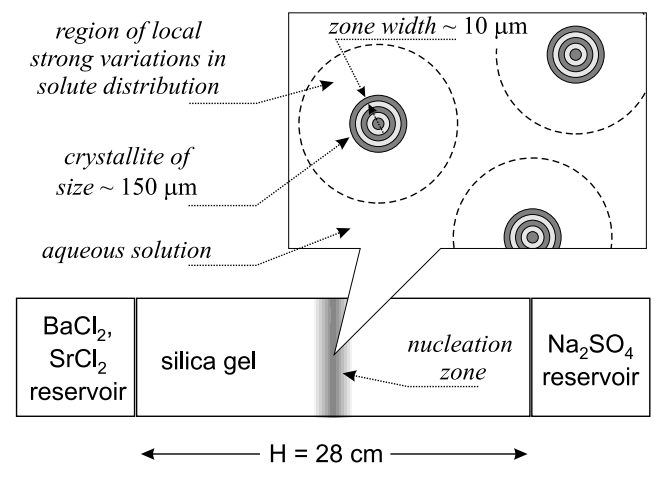

FIG. 1: Experimental setup in which oscillatory zoned crystals of $(\mathrm{Ba}, \mathrm{Sr}) \mathrm{SO}_{4}$ were synthesized in Refs. [3, 4, 5]. The reactants counterdiffuse in the column and (Ba,Sr) $\mathrm{SO}_{4}$ crystals nucleate. The upper window sketches the structure of the nucleation zone and the length scales involved.

The general principle causing $\mathrm{OZ}$ is the autocatalytic or inhibiting interaction of the substrate with the end member concentration in melt or solution [8]. If, for example, the crystal is rich in component $\mathrm{A}$, this will lead to increased growth of this component in an autocatalytic way. Its supply will eventually be limited by diffusion. During this phase the disfavored component $\mathrm{B}$ will accumulate in the solution, leading to a slight increase of B deposition. However, any small increase in B will show autocatalytic effects, whereas the growth of A slowly decreases. The combination of a relatively high $\mathrm{B}$ concentration in combination with the autocatalytically increasing growth rate will then lead to a phase of B dom- 
inated growth. With this, A and B have switched roles and one half cycle is completed. If the interaction of those two processes is interrupted, for example by stirring no OZ will be observed 2].

The specific interaction of autocatalytic growth and component accumulation is subject to the scenario employed and gives rise to different nonlinear schemes. The first quantitative model derived by Haase et al. [9] describes self-organized oscillatory zoning from the melt applying moving boundaries and a generically autocatalytic growth term. In a subsequent series of publications Wang and Merino introduced the boundary layer approximation for the treatment of zoned crystals grown hydrothermally [10], from solution [11], and from melt [12]. Later, non generic growth terms derived from the physics of growth processes from the melt were introduced by L'Heureux [13] using constitutional undercooling and by Wang and Wu [14] employing the excess enthalpy of crystallization.

The most sophisticated models currently available for end member OZ from solution have been developed by L'Heureux et al. [15, 16, 17]. These models apply the boundary layer approximation, as well, and in addition to the otherwise deterministic nature the influence of noise on the onset of $\mathrm{OZ}$ is studied. The non-linear growth term applied is phenomenologically obtained from the local probability to find a matching kink site as proposed by Markov [18]. However, the local nature of this mechanism does not provide the synchronization effects necessary to describe homogeneous growth fronts resulting in the ring like composition oscillations found in the experiment [17].

In this paper we present a boundary reaction diffusion model for OZ in binary solid solutions grown from aqueous solution. We abandon the boundary layer approximation and explicitly treat the diffusion above the crystal without further approximations. Furthermore, the growth rate, being the central ingredient of every model, is derived directly from considerations of the physical growth mechanisms. We apply the concept of layer-bylayer growth under continuous generation of new steps which is, e.g., relevant for growth by screw dislocations or $2 \mathrm{D}$ nucleation. The growth mechanism results as an interplay of different processes including bulk diffusion, adsorption, surface diffusion, and eventually desorption or incorporation into the crystal. The non-linearity necessary to generate OZ is obtained by the compositiondependence of the mean life time of adatoms in the adsorbed layer or, equivalently, the interaction of adatoms with the crystal surface.

In Sect. II the different aspects of the model are introduced. The resulting model equations are summarized in Sect. III. Then, Sect. IV analyses this model close to the stationary point. Sect. V presents its numerical analysis, including all nonlinear effects. Finally, the obtained results are discussed in Sect. VI and concluded in Sect. VII.

\section{PHYSICAL BACKGROUND}

The model under consideration describes the diffusion processes in the bulk solution, the growth process following from the coupling between crystal and solution, and the evolution of the crystal composition.

\section{A. General}

Based on the slow crystal growth observed in the experiments 3, 4, 5], we apply screw dislocations as the step generating mechanism and describe the subsequent growth process via step advance. Screw dislocation driven growth can cross over to two dimensional nucleation, as shown by Pina et al. [4]. However, this will not affect the validity of the model because the specific process of step generation is not of immediate importance. We assume that after nucleation the crystal surface will reach a steady-state when the density of the step generating islands or spirals does not change any more, because of coalesence of the terraces according to [19]. A refined model would be necessary to take into account any effects related to anisotropic growth as found by Pina et al. [20] or to account for the curvature of small spirals. Since we are interested in the basic mechanisms we refrain from such detailed modelling and just consider infinite step trains which on average are a distance $l$ apart; see Fig. 2 Typically, $l$ is in the nanometer-regime. The coordinates $z$, characterizing the distance from the crystal surface and $x$, orthogonal to the steps, are indicated. This describes a one-dimensional crystal surface which starts at $z=0$, i.e. the total system is a $2 \mathrm{D}$-system.

In general, quantities like the solute concentration $C(x, z)$ depend on $x$ and $z$. Conceptually, the $x$ dependence can be separated into two different contributions. First, there exists a periodic contribution with quasiperiod $l$. It expresses the fact that the concentration close to the steps will be smaller. However, in the limit, considered below, only the concentration, averaged over the length scale of $l$, will enter. Therefore this $x$ dependence of $C(x, z)$ is not relevant. Second, there can be variations on length scales much larger than $l$. Experimentally, it is observed that the growth behavior does not change along the surface of one crystallite of size $150 \mu$ at a given time. Furthermore, a straightforward extension of the stability analysis, presented below, shows that the maximum instability for fluctuations along one crystal surface are for zero wave vector. Thus it is realistic to hope that the leading mechanism of $\mathrm{OZ}$ can be derived from study of the $z$-dependence alone. In the present work, we will therefore neglect a possible long-range $x$ dependence and restrict ourselves to the $1 \mathrm{D}$ model.

In the present paper we deal with a two species model $(i=1,2)$ for the crystal growth from solution (Ba and $\mathrm{Sr}$, respectively), thereby neglecting possible variations of the $\mathrm{SO}_{4}{ }^{2-}$ concentration. This can be justified in two ways. First, using the OZ model by L'Heureux [15] we 


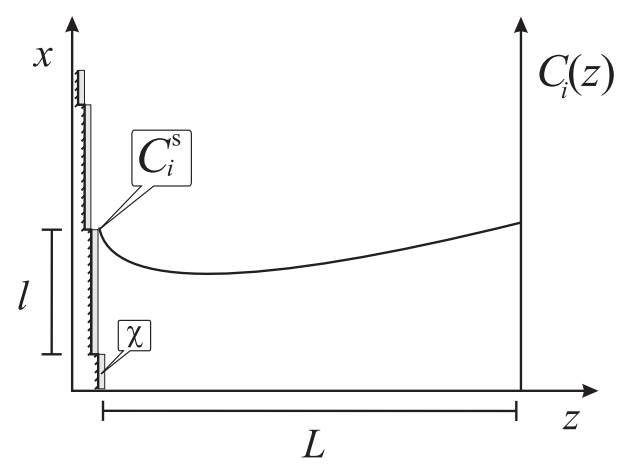

FIG. 2: Scheme of the 2D-model. The concentration is averaged over a sufficiently large $x$-region, giving rise to a $1 \mathrm{D}$ model with a concentration $C(z)$.

have verified that a system with artificially fixed $\mathrm{SO}_{4}{ }^{2-}$ concentration exhibits basically the same dynamics [21]. Second, it describes also the solid state formation for the three component system $\mathrm{SO}_{4}{ }^{2-}, \mathrm{Ba}$, and $\mathrm{Sr}$, studied by Putnis et al. when the concentration of $\mathrm{SO}_{4}{ }^{2-}$ is sufficiently high. The mole fraction of component 1 on the crystal surface is denoted by $\chi(0 \leq \chi \leq 1)$. Throughout this work we assume the same molar volume for both species in the solid phase.

\section{B. Crystal-Solution Interface}

The central element of this model is the coupling term between the crystal growth rate and the surface species concentration. We have used the classical approach by Gilmer et al. 22 which itself builds on the BCF-theory 19]: solute particles adsorb on the crystal surface and diffuse along it. If before desorbing they come into contact with a step on the crystal surface, they are incorporated. If they do not meet a step in time, they desorb and become part of the solution again.

On their path along the crystal surface the adatoms experience many different local environments depending on the crystal composition. The mean energy difference $E_{i}(\chi)$ of the adsorption reflects the interaction with the crystal surface and the solvation process. Within the mean-field description it can be approximated by a linear combination of the different adsorption energies:

$$
\begin{aligned}
& E_{1}(\chi)=\chi E_{11}+(1-\chi) E_{12}-E_{1}^{\text {sol }}, \\
& E_{2}(\chi)=\chi E_{12}+(1-\chi) E_{22}-E_{2}^{\text {sol }} .
\end{aligned}
$$

Here, $E_{i j}=E_{j i}$ is the adsorption energy for component $i$ on a surface formed by component $j$ and $E_{i}^{\text {sol }}$ represents the solvation energy of species $i$.

In the subsequent mathematical treatment it is useful to rewrite expressions (11) in the symmetrized form

$$
\begin{aligned}
& E_{1}(\chi)=2(-\chi \phi+(1-\chi) \theta+\eta) k_{B} T+\Delta E_{\mathrm{m}}^{\mathrm{ad}}, \\
& E_{2}(\chi)=2(\chi \theta+(1-\chi) \phi-\eta) k_{B} T+\Delta E_{\mathrm{m}}^{\mathrm{ad}},
\end{aligned}
$$

where the dimensionless potentials

$$
\begin{aligned}
& \phi=\left(E_{22}-E_{11}\right) / 4 k_{B} T, \\
& \theta=\left(2 E_{12}-\left(E_{11}+E_{22}\right)\right) / 4 k_{B} T, \\
& \eta=\left(E_{2}^{\text {sol }}-E_{1}^{\text {sol }}\right) / 4 k_{B} T .
\end{aligned}
$$

and the mean homogeneous adsorption energy

$$
\Delta E_{\mathrm{m}}^{\mathrm{ad}}=(1 / 2)\left(E_{11}+E_{22}\right)-(1 / 2)\left(E_{1}^{\mathrm{sol}}+E_{2}^{\mathrm{sol}}\right)
$$

have been introduced. Here, $k_{B}$ is the Boltzmann constant and $T$ the temperature. The potential $\phi$ represents the asymmetry between homogeneous adsorption energies, whereas $\theta$ is a measure for the preference of homogeneous over heterogeneous adsorption. We consider the case $0 \leq \phi \leq \theta$. The potential $\phi$ can be assumed to be nonnegative due to symmetry reasons; see Eq. (3). The limit $\phi=\theta$ implies $E_{12}=E_{22}$, so that the crystal growth properties of species 2 are independent of the composition of the crystal surface. The case $\phi>\theta$, corresponding to a different type of crystal growth instability, is beyond the scope of the present paper. The last parameter $\eta$ reflects the solution energy difference of the two types of particles.

For the crystal growth two time scales are of primary importance. First, the inverse of the adsorption time $\tau_{a}$ denotes the rate with which a particle in the solution layer above the crystal surface adsorbs. Thus, $a C_{i}^{s} / \tau_{a}$ is the particle flux on the crystal surface where $a$ is the typical distance between atoms and $C_{i}^{s}=C_{i}(z=0)$ the mean concentration of component $i$ in the solution just above the crystal surface. We assume that $\tau_{a}$ is the same for both species. Second, $\tau_{d, i}$ is the mean residence time of adatoms on the surface. Detailed balance between both time scales requires

$$
\tau_{d, i}(\chi)=\tau_{a} \exp \left[-E_{i}(\chi) / k_{B} T\right] .
$$

Thus the composition-dependence of $\tau_{d, i}$ is due to the composition-dependence of the adsorption potentials $E_{i}(\chi)$. From $\tau_{d, i}$ the mean diffusion length $l_{i}^{\mathrm{s}}$ can be obtained using the Einstein relation

$$
\begin{aligned}
l_{i}^{\mathrm{s}}(\chi) & =\sqrt{D_{s} \cdot \tau_{d, i}(\chi)}, \\
& =\sqrt{D_{s} \cdot \tau_{a}} \exp \left[-\frac{1}{2} E_{i}(\chi) / k_{B} T\right] .
\end{aligned}
$$

The adatom diffusion coefficient $D_{s}$ characterizes the elementary atomic movements of the adatoms on the crystal surface. We also assume that $D_{s}$ is the same for both the species.

By substitution of equation (2) one explicitly obtains for the mean diffusion length

$$
\begin{aligned}
& l_{1}^{\mathrm{s}}(\chi)=l_{D} f_{\eta} \exp [-(-\chi \phi+(1-\chi) \theta)], \\
& l_{2}^{\mathrm{s}}(\chi)=l_{D} f_{\eta}^{-1} \exp [-(\chi \theta+(1-\chi) \phi)]
\end{aligned}
$$

with

$$
l_{D}:=\sqrt{D_{s} \tau_{a}} \exp \left(-\Delta E_{\mathrm{m}}^{\mathrm{ad}} / 2 k_{B} T\right)
$$


and

$$
f_{\eta}:=\exp (-\eta)
$$

Now the partial growth rate $r_{i}$ of species $i$ can be expressed as a combination of the adsorption flux and a success factor $q_{i}$, describing the fraction of adatoms that will actually contribute to crystal growth, whereas the others desorb:

$$
r_{i}\left(\chi, C_{i}^{\mathrm{s}}\right)=\underbrace{\frac{a}{\tau_{a}} \cdot C_{i}^{\mathrm{s}}}_{\text {flux to surface }} \cdot q_{i}(\chi) .
$$

Following Ref. 15 we have neglected a term, representing the equilibrium concentration which is irrelevant under significant supersaturation as present in the experiment. The adsorption of a particle is hindered by the breakup of the solution shell and consequently takes longer than a normal diffusion step in the solution bulk. It is reasonable to assume that the adsorption time scale $\tau_{a}$ is much larger than the typical time scale $a^{2} / D$ of free diffusion in the solution, i.e. $\sqrt{D \tau_{a}} \gg a$. Since $l$ is also a microscopic length scale one may even expect that $\sqrt{D \tau_{a}} \gg l$. Then the location of successive adsorption processes of the same particle are uncorrelated with respect to the position of the steps and this simple probabilistic approach becomes justified.

The growth is supported by surface diffusion from both the sides of a step. In the limit $l_{i}^{\mathrm{s}} \ll l$ the atoms adsorbing within the distance $l_{i}^{\mathrm{s}}$ of a step will typically contribute to crystal growth, whereas all the remaining adatoms will desorb after some time. Therefore, the probability to meet a step is of the order of $l_{i}^{\mathrm{s}} / l$ and can more precisely be expressed as 22

$$
q_{i}(\chi)=\frac{2 l_{i}^{\mathrm{s}}(\chi)}{l} .
$$

For arbitrary ratio $l_{i}^{\mathrm{s}} / l$ one obtains an additional factor $\tanh \left(l / 2 l_{i}^{\mathrm{s}}\right)$ which in the limit $l_{i}^{\mathrm{s}} \ll l$ turns out to be unity 22].

For later convenience we gather the system constants into a characteristic time

$$
\tau=\frac{l \tau_{a}}{2 l_{D}}
$$

and defining

$$
\tau_{i}(\chi)=\tau \frac{l_{D}}{l_{i}^{\mathrm{s}}(\chi)}
$$

the individual growth rates can be written as

$$
r_{i}\left(\chi, C_{i}^{s}\right)=\frac{a C_{i}^{\mathrm{s}}}{\tau_{i}(\chi)} .
$$

Then the resulting continuity relation at the crystal surface reads

$$
\left.D_{i} \frac{\partial C_{i}(z, t)}{\partial z}\right|_{z=0}=r_{i}\left(\chi, C_{i}^{s}\right)
$$

This expresses the particle flux, on the left side, from the solution to the crystal and, on the right side, in the continuous solution close to the surface. An analogous boundary condition has been used in the classical work by Gilmer et al. [22].

\section{Bulk Solution}

The description of the bulk solution follows the following picture: At the beginning of the experiment the components begin to diffuse from the reservoirs into the gel column. As their diffusion profiles begin to overlap close to the middle of the column and the nucleation barrier is overcome, crystallites form. Due to the the narrow nucleation zone [6] these nuclei must act as an effective sink with respect to the current of $\mathrm{Ba}^{2+}, \mathrm{Sr}^{2+}$, and $\mathrm{SO}_{4}{ }^{2-}$ from their reservoirs.

Based on these considerations, this model is set up as a source-sink-system with a gradient in between. In this aspect it differs distinctly from the models proposed by L'Heureux et al. 15, 16, 17], where the crystal is considered to be growing through a homogeneously supersaturated medium. Consequentially an analogous boundary layer approximation cannot be applied to the present system.

Experimentally, the following scales are observed [3]: (i) Growth velocity $V \approx 10^{-8} \mathrm{~cm} / \mathrm{s}$ as estimated from the crystal thickness and the total growth time. (ii) Thickness $H_{o z} \approx 10^{-5} \mathrm{~cm}$ of individual layers. (iii) Time $T_{\mathrm{oz}} \approx H_{\mathrm{oz}} / V \approx 10^{5} \mathrm{~s}$ during which one pattern layer is formed. (iv) Bulk diffusion constant $D \approx 10^{-5} \mathrm{~cm}^{2} / \mathrm{s}$.

¿From these observables two important length scales can be estimated. (i) The length scale $L_{\mathrm{oz}}$ characterizes the spatial variations of the species distribution caused by oscillatory zoning. It is given by $L_{\mathrm{oz}} \approx\left(D T_{\mathrm{oz}}\right)^{0.5} \approx 1 \mathrm{~cm}$ which is much larger than the crystal size. This is consistent with the fact that the growth behavior does not change along one crystal surface, because during times of small change in $\chi$ the information about the local surface concentration can spread over the whole region. Besides, it rationalizes the mean field approach discussed below. (ii) Furthermore, one may wonder to which degree the motion of the solid phase boundary caused by crystal growth can affect the diffusion fields. For small length scales the concentration field is determined by diffusion $\left(\approx(D t)^{0.5}\right)$ whereas for large scales it is determined by the (nearly) constant growth of the phase boundary $(\approx v t)$. The crossover length scale $L_{\mathrm{v}}$ for which both processes are equally relevant can be thus estimated as $L_{\mathrm{v}} \approx D / V$ which for the present situation is close to $10^{3} \mathrm{~cm}$. This scale exceeds the system size by orders of magnitude.

Because of this estimate the effect of the growth induced motion of the crystallites boundaries on the solute diffusion is ignorable and the crystal surface can be regarded as fixed with respect to the mathematical model.

Finally, we choose the external boundary condition 
such that the reservoir is characterized by a constant influx $G_{i}$ of solute into the system at $z=L$ where $L$ is an arbitrary large length scale.

\section{Surface composition evolution}

To complete the model, a governing equation for the evolution of the crystal surface composition is necessary. Assuming a homogeneous distribution of the components throughout the surface, the composition change with the next time increment $d t$ can be expressed as a function of the current composition $\chi$ and the relation of the growth rates $r_{i}$ :

$$
\frac{d \chi}{d t}=a^{2}\left[(1-\chi) \cdot r_{1}\left(\chi, C_{1}^{s}\right)-\chi \cdot r_{2}\left(\chi, C_{2}^{s}\right)\right] .
$$

This relation reflects mass conservation and has already been used in previous work on OZ [15].

\section{THE BOUNDARY-REACTION-DIFFUSION MODEL}

The 1D formulation of the model discussed above can be described as follows. Diffusion of the components $i=$ 1,2 through the solution is considered within the region $z \in[0, L]$ and is described by the equation

$$
\frac{\partial C_{i}(z, t)}{\partial t}=D_{i} \frac{\partial^{2} C_{i}(z, t)}{\partial z^{2}},
$$

where $L$ should be chosen large enough to satisfy $\partial C / \partial t=0$. At the external boundary $z=L$ the influx of both the components is fixed

$$
G_{i}=\left.D_{i} \frac{\partial C_{i}(z, t)}{\partial z}\right|_{z=L} .
$$

At the crystal surface $(z=0)$ the diffusion flux and the rate of the crystal growth are related by (using expressions (14) and (15))

$$
\left.D_{i} \frac{\partial C_{i}(z, t)}{\partial z}\right|_{z=0}=\frac{a C_{i}^{\mathrm{s}}}{\tau_{i}(\chi)} .
$$

The time scales $\tau_{i}$, as defined in Eq.(13), are given by

$$
\begin{aligned}
& \tau_{1}(\chi)=\tau f_{\eta}^{-1} \exp [-\chi(\phi+\theta)+\theta], \\
& \tau_{2}(\chi)=\tau f_{\eta} \exp [\chi(\theta-\phi)+\phi] .
\end{aligned}
$$

The constants $\tau$ and $f_{\eta}$ have been defined in Eqs. (12) and (9), respectively. Finally, for the solid composition evolution one obtains (compare Eq. (16))

$$
\frac{d \chi}{d t}=a^{2}\left[(1-\chi) \frac{a C_{1}^{s}}{\tau_{1}(\chi)}-\chi \frac{a C_{2}^{s}}{\tau_{2}(\chi)}\right] .
$$

In order to complete the description of OZ it is necessary to calculate the resulting structure of the crystal, i.e. $\chi_{\text {crystal }}\left(z_{\text {crystal }}\right)$. For this purpose we introduce $\zeta(t)$ as the location of the crystal surface at time $t$ in a coordinate system which does not move with the crystal surface. Defining $t_{z}$ as the time for which $\zeta(t)=z_{\text {crystal }}$ one has

$$
\chi_{\text {crystal }}\left(z_{\text {crystal }}\right)=\chi\left(t_{z}\right) .
$$

The function $\zeta(t)$ can be easily obtained from

$$
d \zeta / d t=a^{3}\left[\frac{a C_{1}^{s}}{\tau_{1}(\chi)}+\frac{a C_{2}^{s}}{\tau_{2}(\chi)}\right],
$$

where the left side can be interpreted as the timedependent growth velocity proportional to the cumulative species flux at the surface.

\section{STABILITY ANALYSIS}

The following two sections analyze the behavior of the crystal growth within the boundary-reaction diffusion model specified in the previous one. Particularly we study the crystal growth properties close to the stationary point by means of linear stability analysis.

\section{A. Stationary solution}

Both, Eqs. (17) and (21) describe the time-dependence of the underlying system. For each equation and for fixed $\chi$ the disappearance of the time-derivative yields some ratio $C_{1}^{s} / C_{2}^{s}$, respectively.

Evidently, $(d / d t) \chi(t)=0$ implies (using $f_{\eta}^{2} e^{\phi}=1$ for simplicity)

$$
\frac{C_{1}^{s}}{C_{2}^{s}}=\frac{\chi}{1-\chi} \frac{\tau_{1}(\chi)}{\tau_{2}(\chi)}=\frac{\chi}{1-\chi} \exp (\theta(1-2 \chi)) \equiv N_{\chi}(\chi) .
$$

This nullcline $N_{\chi}(\chi)$ is shown in Fig. [3 It possesses a decreasing branch located in the region $\chi \in\left(\chi_{-}, \chi_{+}\right)$

$$
\chi_{ \pm}:=\frac{1}{2}\left(1 \pm \sqrt{\frac{\theta-2}{\theta}}\right),
$$

when the parameter $\theta$ exceeds the critical value $\theta_{c}=2$. For $\theta<2$ the function $N_{\chi}(\chi)$ is monotonously increasing. In the stationary case the presence of a decreasing branch implies that different values of $\chi$ are associated to the same $C_{1}^{s} / C_{2}^{s}$.

A stationary solution of the diffusion equation Eq. (17), fulfilling the boundary conditions Eqs. (19), reads

$$
C_{i}(z)=\frac{G_{i} \tau_{i}(\chi)}{a}+\frac{G_{i}}{D_{i}} z
$$

giving the surface concentration

$$
C_{i}^{s}=\frac{G_{i} \tau_{i}(\chi)}{a} .
$$




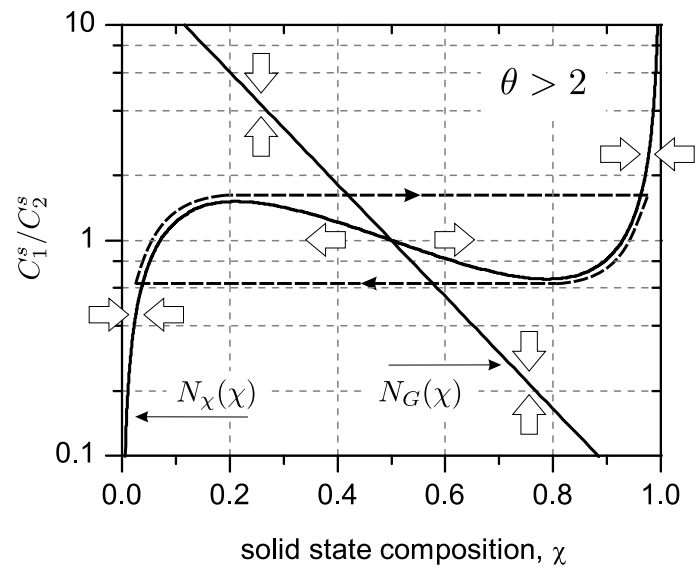

FIG. 3: Stationary functions $N_{\chi}(\chi)$ and $N_{G}(\chi)$ vs. the solid state composition $\chi$. The chosen parameters are $\theta=3$, $f_{\eta}^{2} e^{\phi}=1$, and $G_{1}=G_{2}$. For this choice the stationary value $\chi^{s t}$ is 0.5 . The dashed curve illustrates the typical construction of the limit cycle for the developed instability.

This yields (using again $f_{\eta}^{2} e^{\phi}=1$ )

$$
\frac{C_{1}^{s}}{C_{2}^{s}}=\frac{G_{1}}{G_{2}} \frac{\tau_{1}(\chi)}{\tau_{2}(\chi)}=\frac{G_{1}}{G_{2}} \exp (\theta(1-2 \chi)) \equiv N_{G}(\chi)
$$

in the stationary limit. The function $N_{G}(\chi)$ is also shown in Fig. 3. Both the functions $N_{\chi}(\chi)$ and $N_{G}(\chi)$ intersect at $\chi=\chi^{\text {st }}$ given by

$$
\chi^{\mathrm{st}}=\frac{G_{1}}{G_{1}+G_{2}} \equiv \frac{G_{1}}{G}
$$

thereby defining the total incoming flux $G=G_{1}+G_{2}$. It defines the stationary point of the present system for which both Eq. (17) and Eq. (21) have vanishing timederivatives.

Now we discuss some immediate consequences of the properties of the functions $N_{\chi}(\chi)$ and $N_{G}(\chi)$. For this purpose we consider the $\left(\chi, C_{1}^{s} / C_{2}^{s}\right)$-plane. These functions separate different regions of the phase plane. Naturally, a point on this plane does not describe the complete system configuration because the diffusion is reduced to $C(z=0)$. For $C_{1}^{s} / C_{2}^{s}>N_{\chi}(\chi)$ the time derivative of $\chi(t)$ is positive and vice versa. This means that a system above $N_{\chi}(\chi)$ is driven toward larger $\chi$, whereas a system below behaves oppositely.

In case of a standard relaxation oscillator the equation for $\dot{\chi}$ would be complemented by an equation for $(d / d t)\left(C_{1}^{s} / C_{2}^{s}\right)$. Such an equation does not exist for the present model. However, an implicit time evolution of $\left(C_{1}^{s} / C_{2}^{s}\right)$ is expressed by Eq. (28). When $(d / d t) \chi(t)$ is very small the diffusion field can adjust to the boundary conditions. This way the ratio $C_{1}^{s} / C_{2}^{s}$ will be adjusted until Eq. (28) is fulfilled. In particular, $C_{1}^{s} / C_{2}^{s}$ will decrease for $\left(C_{1}^{s} / C_{2}^{s}\right)>N_{G}(\chi)$ and vice versa. This is reflected by the arrows in Fig 3
The simplest form of a possible time evolution giving rise to oscillatory behavior is shown in Fig. 3] If there is a time-scale separation between the $\chi$-variations and the variations of the surface concentrations $C_{i}^{s}$ the system would move from the left maximum of $N_{\chi}(\chi)$ to the right until $\dot{\chi} \approx(d / d t)\left(C_{1}^{s} / C_{2}^{s}\right)$. Then the system has time to adapt $\left(C_{1}^{s} / C_{2}^{s}\right)$ to the present value of $\chi$ thereby moving down along the $N_{\chi}(\chi)$-curve. Once the the minimum is reached $(d / d t)\left(C_{1}^{s} / C_{2}^{s}\right)$ will drive the trajectory away from $N_{\chi}(\chi)$ quickly resulting in $|\dot{\chi}| \gg\left|(d / d t)\left(C_{1}^{s} / C_{2}^{s}\right)\right|$ which will move it towards the left branch of $N_{\chi}(\chi)$. Then the second half of the oscillation may start. As will be shown below via numerical simulations the actual behavior is somewhat different. In any event, the general possibility of oscillatory behavior requires a nonmonotonous behavior of $N_{\chi}(\chi)$, i.e. $\theta>\theta_{c}=2$. However, the linear stability analysis will reveal that this condition is not sufficient.

\section{B. Linear stability analysis}

The stability of the stationary crystal growth is analyzed with respect to infinitesimal perturbations of the solute distribution in the solution and the solid state composition around $\chi=\chi^{\text {st }}$ and the corresponding values of $C_{i}^{s}$, given by Eq. (27) which will be denoted $C_{i}^{\text {st }}$. We choose

$$
\delta C_{i}(z, t)=\delta C_{i}^{s} \exp \left(\gamma t-p_{i} z\right)
$$

and

$$
\delta \chi(t)=\delta \chi \exp (\gamma t) .
$$

Here $\delta C_{i}^{s}$ and $\delta \chi$ are the amplitudes whereas the complex wave number $p_{i}=\operatorname{Re} p_{i}+\mathrm{i} \operatorname{Im} p_{i}$ describes the decay of the concentration perturbations above the crystal surface. It requires $\operatorname{Re} p_{i}>0$. The instability arises when the real part of the perturbation increment $\gamma$ becomes positive. The chosen form (30) is compatible with the time-evolution close to the stationary point.

The infinitesimal form of Eq. (21) is of primary interest. A short calculation gives

$$
\frac{\gamma}{a^{2} G}=-1+\chi^{\mathrm{st}}\left(1-\chi^{\mathrm{st}}\right)\left[2 \theta+\frac{\delta C_{1}}{C_{1}^{\mathrm{st}} \delta \chi}-\frac{\delta C_{2}}{C_{2}^{\mathrm{st}} \delta \chi}\right] .
$$

The relevant ingredient $\delta C_{i}^{s} /\left(C_{i}^{\text {st }} \delta \chi\right)$ can be obtained from the boundary condition (19) as

$$
\frac{\delta C_{i}^{s}}{C_{i}^{\mathrm{st}} \delta \chi}=\frac{a \partial \ln \tau_{i} / \partial \chi}{a+D_{i} p_{i} \tau_{i}\left(\chi^{\mathrm{st}}\right)} .
$$

Finally, from Eq. (17) one has

$$
\gamma=D_{1} p_{1}^{2}=D_{2} p_{2}^{2},
$$


i.e. the relation between the $p_{i}$ and $\gamma$. Combining Eqs. (31), (32) and (33) and using the specific dependencies (20) we finally get

$$
\begin{aligned}
& \frac{\gamma}{a^{2} G}=-1+\chi^{\mathrm{st}}\left(1-\chi^{\mathrm{st}}\right) \\
& \quad \times\left[(\theta+\phi) \frac{D_{1} \tau_{1} p_{1}}{D_{1} \tau_{1} p_{1}+a}+(\theta-\phi) \frac{D_{2} \tau_{2} p_{2}}{D_{2} \tau_{2} p_{2}+a}\right] .
\end{aligned}
$$

How does the nature of the stability depend on the total flux $G$ ? One always has $C_{i}^{\text {st }} \propto G$. Furthermore, in the limit of large $G$ one also obtains $\gamma \propto G$ and, using Eq. (33), $p_{i} \propto \sqrt{G}$. As a consequence one has $\delta C_{i} \propto$ $C_{i} / p_{i} \propto \sqrt{G}$. Thus, Eq. (31) boils down to

$$
\gamma=a^{2} G\left[-1+\chi^{\mathrm{st}}\left(1-\chi^{\mathrm{st}}\right) 2 \theta\right] .
$$

One has $\gamma>0$ exactly when $\chi^{\text {st }} \in\left(\chi_{-}, \chi_{+}\right)$, i.e. $\chi^{\text {st }}$ is on the unstable branch of $N_{\chi}(\chi)$. In contrast, for $G \rightarrow 0$ one has $\gamma=-a^{2} G<0$. This can be seen from Eq. (34) because in this limit also $p_{i} \rightarrow 0$. Thus there exists a critical flux $G_{c}$ such that $\operatorname{Re} \gamma=0$ for $G=G_{c}$ and $\operatorname{Re} \gamma>$ 0 for $G>G_{c}$. Since $\gamma=p_{i}=0$ cannot be a solution from Eq. (34) the disappearance of $\operatorname{Re} \gamma$ implies that $\gamma$ is purely imaginary.

Our goal is, first, to determine $G_{c}$ explicitly and, second, to understand its dependence on the model parameters on a more qualitative level, i.e. $G_{c}=G_{c}(\chi)$ $\theta, \phi, \ldots)$.

\section{Exact solution}

From Eq. (34) the critical value $G_{c}$ can be determined. As shown in the Appendix the critical value $G_{c}$ in parametric form is given by the expression

$$
\begin{aligned}
G_{c} & =\frac{1}{\sqrt{D_{1} D_{2}} \tau^{2}} \frac{e^{-\phi(1-2 \chi)-\theta} \zeta}{\sqrt{2} \chi(1-\chi)} \\
& \times\left[\frac{(\theta+\phi) \Delta}{(\sqrt{2} \zeta \Delta+1)^{2}+1}+\frac{(\theta-\phi) / \Delta}{(\sqrt{2} \zeta / \Delta+1)^{2}+1}\right]^{-1} .
\end{aligned}
$$

Here the variable $\zeta$ is the root of the equation

$$
(\theta+\phi) \Psi(\sqrt{2} \zeta \Delta)+(\theta-\phi) \Psi(\sqrt{2} \zeta / \Delta)=\frac{1}{\chi(1-\chi)},
$$

where the function $\Psi(x)$ is defined as

$$
\Psi(x)=\frac{x(x+1)}{(x+1)^{2}+1}
$$

and the function $\Delta(\chi)$ is

$$
\Delta(\chi)=\Delta_{0.5} e^{\theta(1-2 \chi) / 2}
$$

with the prefactor $\Delta_{0.5}$ given by the expression

$$
\Delta_{0.5}^{2}=\sqrt{\frac{D_{1}}{D_{2}}} f_{\eta}^{-2} e^{-\phi} .
$$

Since $\phi \leq \theta$, both terms on the left-hand side of Eq. (37) are non-negative increasing functions of the argument $\zeta$. The maximum of their sum is equal to $2 \theta$ whereas the minimum of the right-hand side at $\chi=0.5$ is equal to 4 . So, we obtain again the condition $\theta>\theta_{c}$ necessary for instable behavior. In this case for $2 \theta \chi(1-\chi)>$ 1 equation (37) possesses only one root which together with (36) determine the lower boundary $G_{c}$ of the instability region. In the close vicinity of the threshold, $0<2 \theta \chi(1-\chi)-1 \ll 1($ for $\chi \approx 0.5$ ) this equation can be solved analytically, giving the expression

$$
\zeta \approx \frac{\left[(\theta+\phi) / \Delta_{0.5}+(\theta-\phi) \Delta_{0.5}\right]}{4 \sqrt{2}[2 \theta \chi(1-\chi)-1]}
$$

and thus

$$
G_{c} \approx \frac{e^{-2}}{32 \sqrt{D_{1} D_{2}} \tau^{2}} \frac{\left[(\theta+\phi) / \Delta_{0.5}+(\theta-\phi) \Delta_{0.5}\right]^{2}}{[2 \theta \chi(1-\chi)-1]^{3}} .
$$

For the system with $\phi=0$ the asymmetry of the solubilities substantially increases the critical diffusion flux $G_{c}$. Indeed, since the species diffusivities in solutions are typically of the same order, $D_{1} \sim D_{2}$, the species solubility difference reflected in the coefficient $f_{\eta} \ll 1$ or $f_{\eta} \gg 1$ matches (see Eq. (40)) the inequality $\Delta_{0.5} \gg 1$ or $\Delta_{0.5} \ll 1$, respectively. For $\phi \approx \theta$ the term $(\theta-\phi) \Delta_{0.5}$ in (42) can be neglected and an increase of $\Delta_{0.5}$ gives rise to a remarkable decrease in $G_{c}$.

Equation (37) was solved numerically to analyze the system behavior far from the threshold $\theta_{c}=2$. For $\theta=3$ the results for the $\chi$-dependence of $G_{c}$ are presented in Fig. 4. In addition we have included $N_{\chi}(\chi)$ in Fig. 目 The upper frame exhibits the results for $\phi=0$, whereas the lower one contains the strongly asymmetrical case with $\phi=\theta=3$.

The symmetrical system exhibits minimal $G_{c}$ when the species have the same solubility. A difference in solubility as reflected by the change of $\Delta_{0.5}$ by a factor of ten causes $G_{c}$ to increase by a similar factor. For such $\Delta_{0.5}$ the $G_{c}(\chi)$-curves become asymmetrical with respect to $\chi=$ 0.5. Exactly this case should be characterized by the instability forming the limit cycle constructed in Fig. 3 following the standard notions of relaxation oscillations.

The system behavior for $\phi \sim \theta$ is distinctly different as can be seen in the lower fragment of Fig. 团 In particular, for $\phi=3$ a ten-fold increase in $\Delta_{0.5}$ induces a hundredfold drop in $G_{c}$. It should be noted that $\Delta_{0.5}=1$ corresponds to $\eta=\phi / 2$ which is non-zero here. Furthermore, for $\Delta_{0.5}=10$ the dependence $G_{c}(\chi)$ is highly asymmetrical and passes many orders of magnitude. For large values of $\Delta_{0.5}$, the left part of the decreasing branch of $N_{\chi}(\chi)$ can be unstable whereas the right half can be stable. In this case the limit cycle of the developed oscillations deviates substantially from the classical form. 


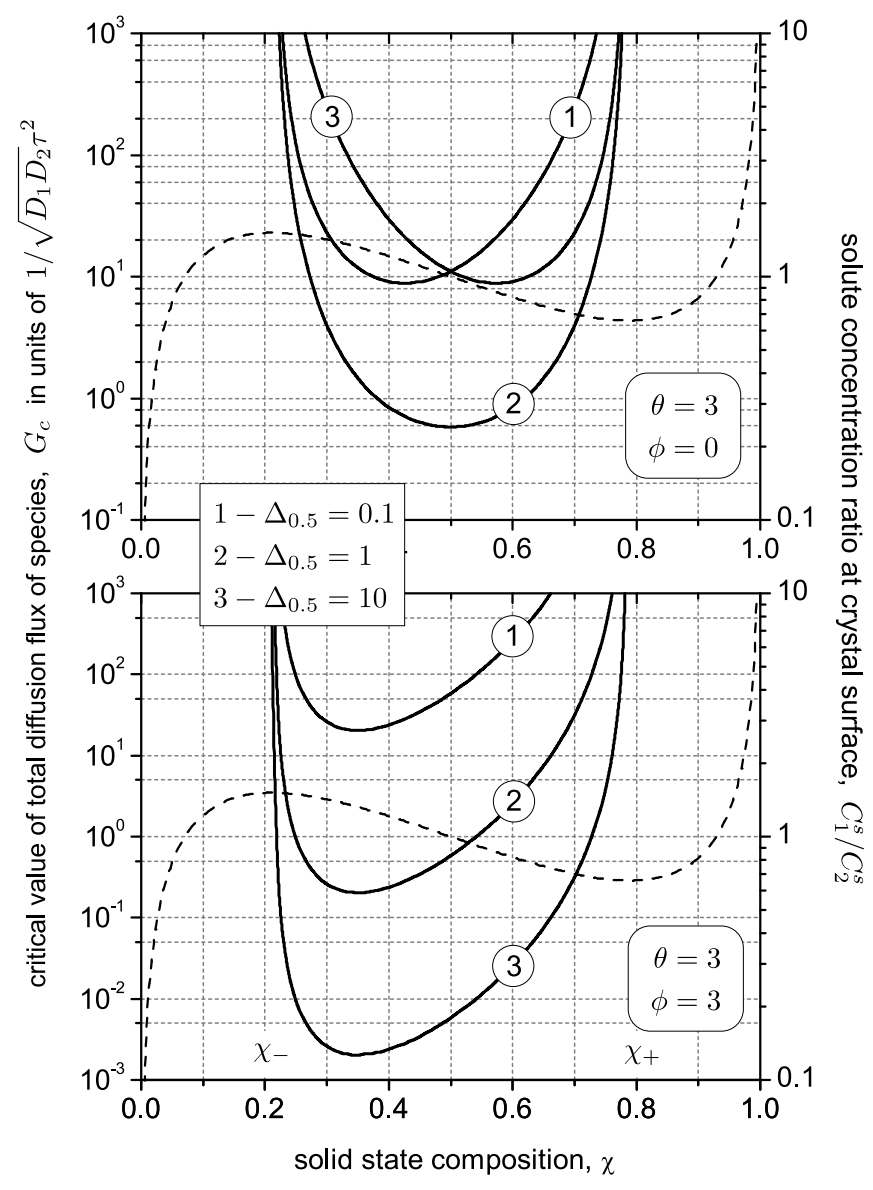

FIG. 4: The critical value of $G_{c}$ vs. $\chi$ for $\theta=3$ and some different $\Delta_{0.5}$ representing the difference in the species solubilities. The upper frame corresponds to a symmetric system with $\phi=0$, the lower one to a strongly asymmetrical one with $\phi=3$. The dashed curves correspond to the function $N_{\chi}(\chi)$.

\section{Instability mechanism: qualitative description}

It is possible to obtain a better understanding of how the degree of instability depends on the system parameters. We use sufficiently large $G$ such that $a \ll\left|D_{i} p_{i} \tau_{i}\right|$. The values of $\tau_{i}$ are always analyzed at $\chi=\chi^{\mathrm{st}}$. For reasons of simplicity we also assume $D_{1}=D_{2}=D$ (implying $p \equiv p_{1}=p_{2}$ ). Then we can rewrite Eq. (32) as

$$
\frac{\delta C_{i}^{s}}{C_{i}^{\text {st }} \delta \chi}=\frac{a}{p D} \frac{\partial \ln \tau_{i} / \partial \chi}{\tau_{i}}
$$

thereby

$$
\frac{\delta C_{1}^{s}}{C_{1}^{\mathrm{st}} \delta \chi}-\frac{\delta C_{2}^{s}}{C_{2}^{\mathrm{st}} \delta \chi}=\frac{a}{p D}\left[-\frac{\theta}{\tau_{1}}-\frac{\theta}{\tau_{2}}-\frac{\phi}{\tau_{1}}+\frac{\phi}{\tau_{2}}\right] .
$$

The cumulative effect of these terms gives rise to a decrease of the real part of $\gamma$. Thus, concentration fluctuations always tend to stabilize the stationary point. It is interesting to analyze the impact of the asymmetry $\phi$. In what follows we will restrict ourselves to the case $\phi=\theta$, where $\delta C_{2}=0$ and the damping is due to the concentration fluctuations of species 1 . Changing from $\phi=0$ to $\phi=\theta$ the relevant term in Eq. (31), characterizing the concentration fluctuations and thus the reduction of the real part of $\gamma$ changes from $-\left(1 / \tau_{1}+1 / \tau_{2}\right)$ to $-2 / \tau_{1}$ which corresponds to a change by a factor of $2 /\left(1+\tau_{1} / \tau_{2}\right)$. Note that

$$
\tau_{1} / \tau_{2}=\Delta_{0.5}^{2} \exp \left[\theta\left(1-2 \chi^{\mathrm{st}}\right)\right]
$$

When $\Delta_{0.5} \gg 1$ the result of the concentration fluctuations is thus reduced which has a positive cumulative effect on the instability onset and, as a result, $G_{c}$ should be decreased. Moreover, this ratio increases as $\chi$ decreases. This rationalizes the asymmetry of the $G_{c}(\chi)$ dependence with smaller values located on the left-hand side. Exactly these features emerge from the solution of the exact equation (34).

\section{NUMERICAL ANALYSIS OF THE NONLINEAR DYNAMICS}

When the growth becomes unstable nonstationary patterns in the solution and the induced pattern in the crystal bulk develop. In order to analyze their characteristic properties the system of equations in Sect. [II] was studied numerically. First, we introduced the spatial and temporal scales $l_{\mathrm{sc}}$ and $\tau_{\mathrm{sc}}$

$$
l_{\mathrm{sc}}=\left(\frac{\sqrt{D_{1} D_{2}} \tau}{a^{2}}\right) a, \quad \tau_{\mathrm{sc}}=\left(\frac{\sqrt{D_{1} D_{2}} \tau}{a^{2}}\right) \tau,
$$

and, in addition, a parameter having the dimension of concentration

$$
C_{\mathrm{sc}}=\frac{1}{a \sqrt{D_{1} D_{2}} \tau} .
$$

Then we rescaled time $t$ and spatial coordinate $z$ as well as the species concentrations $C_{i}$ in these units

$$
t \rightarrow \tau_{\mathrm{sc}} \cdot t, \quad z \rightarrow l_{\mathrm{sc}} \cdot z, \quad C_{i} \rightarrow C_{\mathrm{sc}} \cdot C_{i}
$$

For the sake of simplicity we have kept the same designations for these variables. The fluxes $G_{i}, G$ were also measured in units of

$$
G_{\mathrm{sc}}=\frac{1}{\sqrt{D_{1} D_{2}} \tau^{2}}
$$

namely,

$$
\left(G, G_{i}\right) \rightarrow G_{\mathrm{sc}} \cdot\left(G, G_{i}\right)
$$

Then the obtained system of dimensionless equations was integrated using the Crank-Nicholson method. The temporal and spatial steps of integration as well as the system 
size $L$ were chosen such that the dynamics be practically independent of their particular values.

The following four specific cases were analyzed to demonstrate characteristic features of the physical system. The first two ones are the symmetrical model with parameters $\theta=3, \phi=0, \Delta_{0.5}=1$ and $\chi_{\mathrm{st}}=0.5$ at the initial stage. They differ in the total diffusion flux $G=1$ and $G=50$. The case with $G=1$ describes the system dynamics not too far from the instability boundary $G_{c} \approx 0.6$ (see Fig. 4). Under these conditions the instability was expected to demonstrate quasiharmonic behavior. The case $G=50$ corresponds to the substantially nonlinear stage of the instability.

The other pair of cases are the asymmetrical model with $\theta=3$ and $\phi=3$ being actually a limit situation that can be considered accurately within the present analysis. To single out the effects caused by nonzero values of $\phi$ we restrict ourselves to $\Delta_{0.5}=1$ and $\chi_{\text {st }}=0.3$. Correspondingly, $G_{c}(\chi)$ is minimal for this value of $\chi$. The values of $G$ are set to 0.5 and 5 .

Figure 5 visualizes the surface dynamics for the first two cases. As seen in the frames of its left column, the oscillations of $C_{i}^{s}$ and $\chi$ are rather harmonic as expected. The resulting limit cycle is shown in the lower left frame. Again, as expected, this cycle is of elliptical form located along the unstable branch of the nullcline $N_{\chi}(\chi)$. Even for $G=1$ which is close to $G_{c} \approx 0.6$ the oscillations are already determined by the nullcline.

For $G=50$ the dynamics become relaxation-like and $\chi$ plays the role of the fast variable. As seen in the right column of Fig. 5 the time pattern $\chi(t)$ consists of a sequence of slow motion fragments joined by rather sharp jumps. In agreement with classical relaxation oscillations the fragments of slow dynamics correspond to the system motion along stable branches of $N_{\chi}(\chi)$ whereas the sharp jumps describe the fast transitions between these branches. However, the surface species concentrations exhibit anomalous behavior from the standpoint of classic relaxation oscillations. Most importantly, $C_{1}^{s} / C_{2}^{s}$ display some remarkable variations during the fast dynamics phase of $\chi$. This is still reminiscent of the result of the linear stability analysis where $\delta C_{i}^{s} \propto \delta \chi$. As a result the lines of the limit cycle connecting the increasing branches of the nullcline $N_{\chi}(\chi)$ are not horizontal but inclined to the $\chi$-axis at a certain visible angle. It should be noted that this inclination is not a standard consequence of the finite ratio between the time scales of fast and slow dynamics because, otherwise, the form of these curves would deviate form the straight lines substantially. It is due to the existence of the simultaneous change in both the species concentrations during the period of fast motion.

The asymmetrical system with $\theta=3, \phi=3$ is depicted in Fig. 6 for $G=0.5$ and $G=5$. The basic feature distinguishing this system from the symmetrical one is the strong asymmetry of the critical diffusion flux with respect to $\chi=0.5$. In particular, as shown in Fig. 4 for $G=0.5$ only the left half of the decreasing branch of the nullcline $N_{\chi}(\chi)$ is unstable whereas for $G=5$ the instability region is located approximately within the boundaries $0.2<\chi<0.6$. The resulting limit cycles are shown in the lower frames of Fig. [6 For $G=0.5$ the stationary point is not too far from the instability boundary and the limit cycle is located in a rather narrow neighborhood of the unstable branch of $N_{\chi}(\chi)$. For $G=5$ the separation of the system dynamics into the slow and fast motion fragments becomes pronounced. In this case the limit cycle embraces even an increasing branch of the $N_{\chi}(\chi)$. However, its lower part after passing the minimum near $\chi=0.8$ continues to follow the formerly unstable fragment of the decreasing nullcline branch until it reaches the instability boundary at $\chi \approx 0.6$. Only after passing this point it leaves the branch and jumps to the opposite stable branch of $N_{\chi}(\chi)$. This effect of "adhesion" to the unstable branch of $N_{\chi}(\chi)$ is of another nature than the well known "French duck" fragment of the limit cycle for standard relaxation oscillations (see, e.g., Ref. [27]), caused by the close proximity of the stationary point to the extreme of the nullcline.

The two time patterns of the observed oscillations deviate substantially in shape both from the quasiharmonic oscillations and relaxation oscillations. Especially for the flux $G=5$ the found pattern looks like a sequence of pronounced spikes joined by fragments of slow motion along the left decreasing branch of $N_{\chi}(\chi)$.

The resulting crystal profiles are shown in Fig. 7 Basically, the profiles are scaled mirror images of the timedependent $\chi$ as shown in Figs. 5 and $[6$ because the growth velocity only weakly varies with time. As should be expected the spatial oscillation period is orders or magnitude larger than the atomic scale.

\section{DISCUSSION}

We have presented a 1D model for OZ. The growth rate as the central non-linear coupling term between the solution and the crystal was derived based on layer-bylayer crystal growth mechanisms. For this purpose particle adsorption, surface diffusion and finally desorption or inclusion processes at the steps are taken into account. This way, adsorbed particles do not only experience the local environment of their adsorption site, but a much more averaged one depending strongly on the composition of the crystal surface. This is an essential feature of the present model, because together with volumetric species diffusion it may provide the synchronization effect necessary to successfully describe the experimental findings in more than one dimension.

Our model (Eqs.(17)-(21) ) differs in several respects from the existing model of L'Heureux et al. [15, 16]. (i) The most essential difference is the choice of the growth mechanism, i.e. the definition of the growth rate $r_{i}(\chi)$. In Refs. [15, 16] a phenomenological equation based on local atom adsorption [18] is formulated. It is one of the simplest polynomial expression for $r_{i}(\chi)$ which displays 

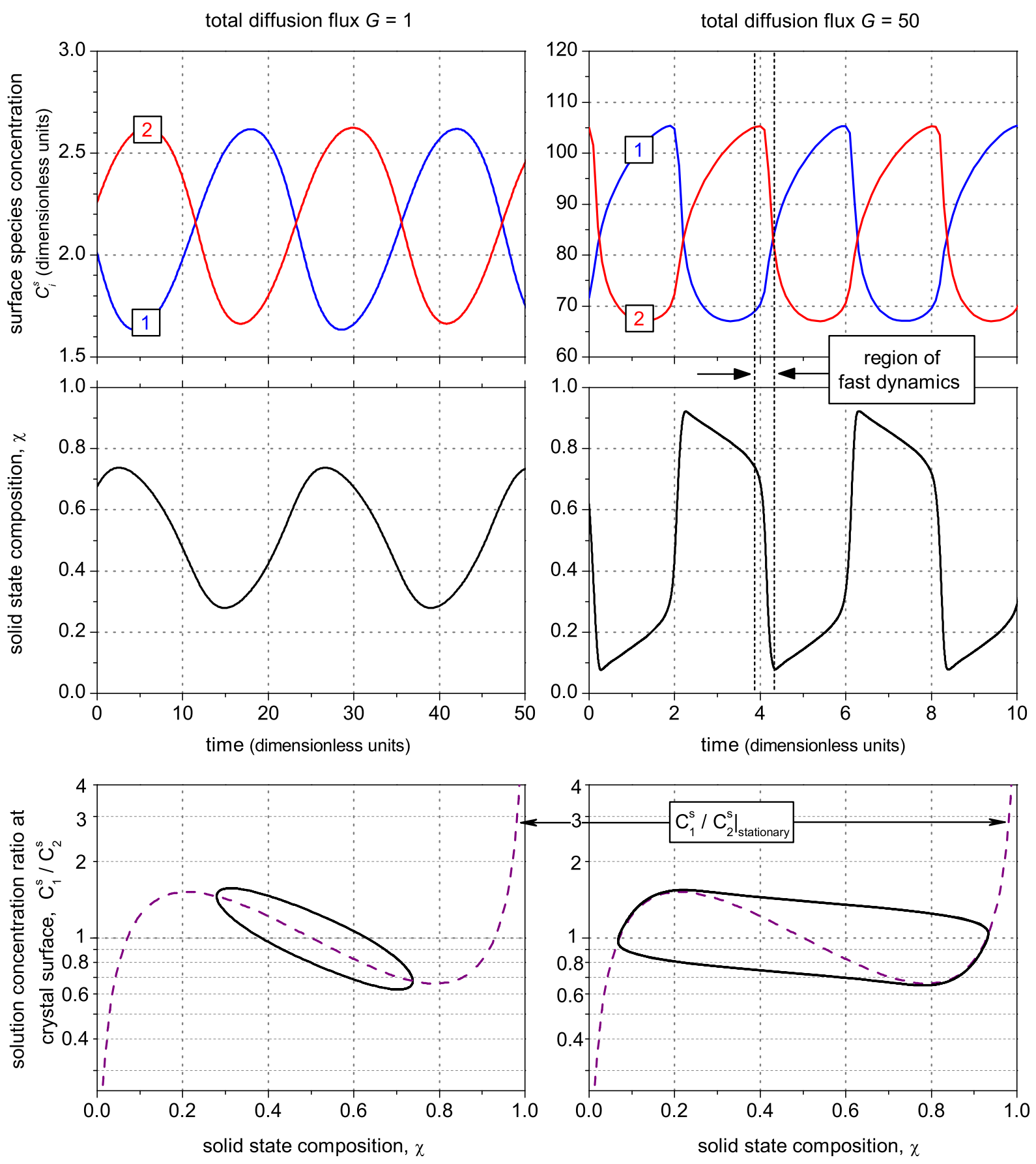

FIG. 5: Dynamics of the symmetrical system $\theta=3, \phi=0, \Delta_{0.5}=1, \chi_{\mathrm{st}}=0.5$. The left column has the results obtained for $G=1$ which is larger than $G_{c}=0.6$, see Fig. 团 The right column exhibits the results for $G=50$.

nonlinear behavior. (ii) The boundary condition at $z=0$ used in 15, 16] for crystal growth from solution can be written as

$$
D_{i} \partial C_{i} / \partial z=\chi_{i}\left(r_{1}+r_{2}\right)
$$

in our notation. Here one uses $\chi_{1}=1-\chi_{2}=\chi$. It is identical to Eq. (19) if $(\mathrm{d} / \mathrm{d} t) \chi(t)=0$. But as soon as $\chi$ changes with time both boundary conditions are not identical. Actually, to derive Eq. (49) one can formulate the mass balance in a newly generated crystal layer. Then Eq. (49) only emerges if the $\chi$-value in the layer at some time, determining the nature of the growth rates $r_{i}(\chi)$ during the growth of the new layer is identical to the $\chi$-value in the resulting new layer. In general this is not the case. Thus we feel that Eq. (19) is more generally valid although from a practical point of view no 

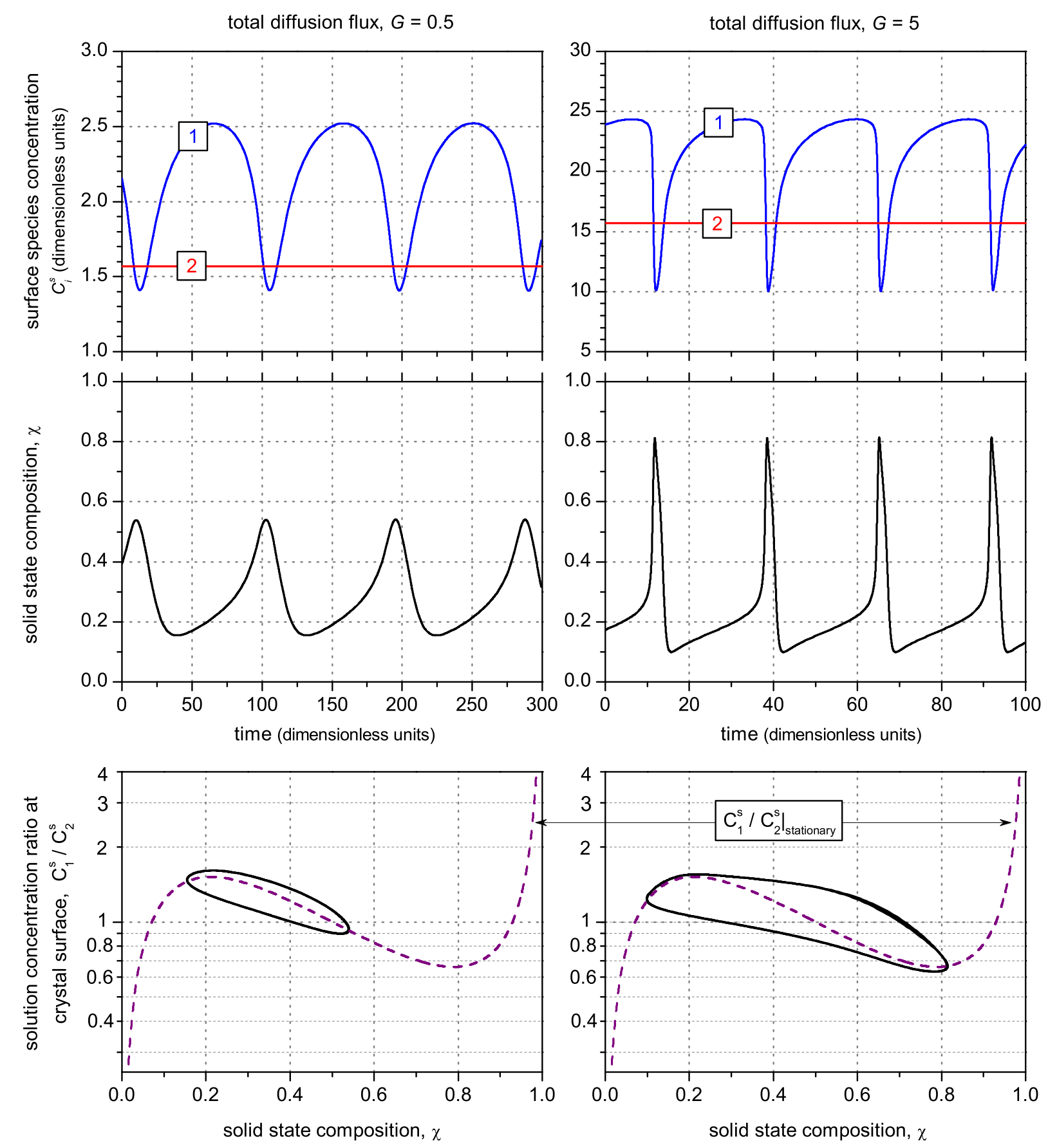

FIG. 6: Dynamics for the asymmetrical system $\theta=3, \phi=3, \Delta_{0.5}=1, \chi_{\mathrm{st}}=0.3$. The left column presents the data obtained for the total diffusion flux $G=0.5$, whereas the right one exhibits the same data for $G=5$.

essential difference should be present. (iii) We specify the gradient at $z=L$ whereas in [15, 16] the concentration is given. Our choice is conceptually more simple because the value of $L$ does not enter the calculations (see e.g. Eq. (260). However, the underlying physical picture does not change whether the gradient or the concentration itself are fixed as long as $L$ is sufficiently large. (iv) Another distinctive difference is the explicit calculation of the diffusion field in the solution above the crystal. We do not apply the boundary layer approximation, regarding a crystal growing through a supersaturated solution [15], but we neglect the spatial growth with respect to the diffusion. This way, we obtain a source-sink system with a diffusion field that has to be treated explicitly, because the interaction of particle accumulation or depletion on the solution side and the autocatalytic growth 

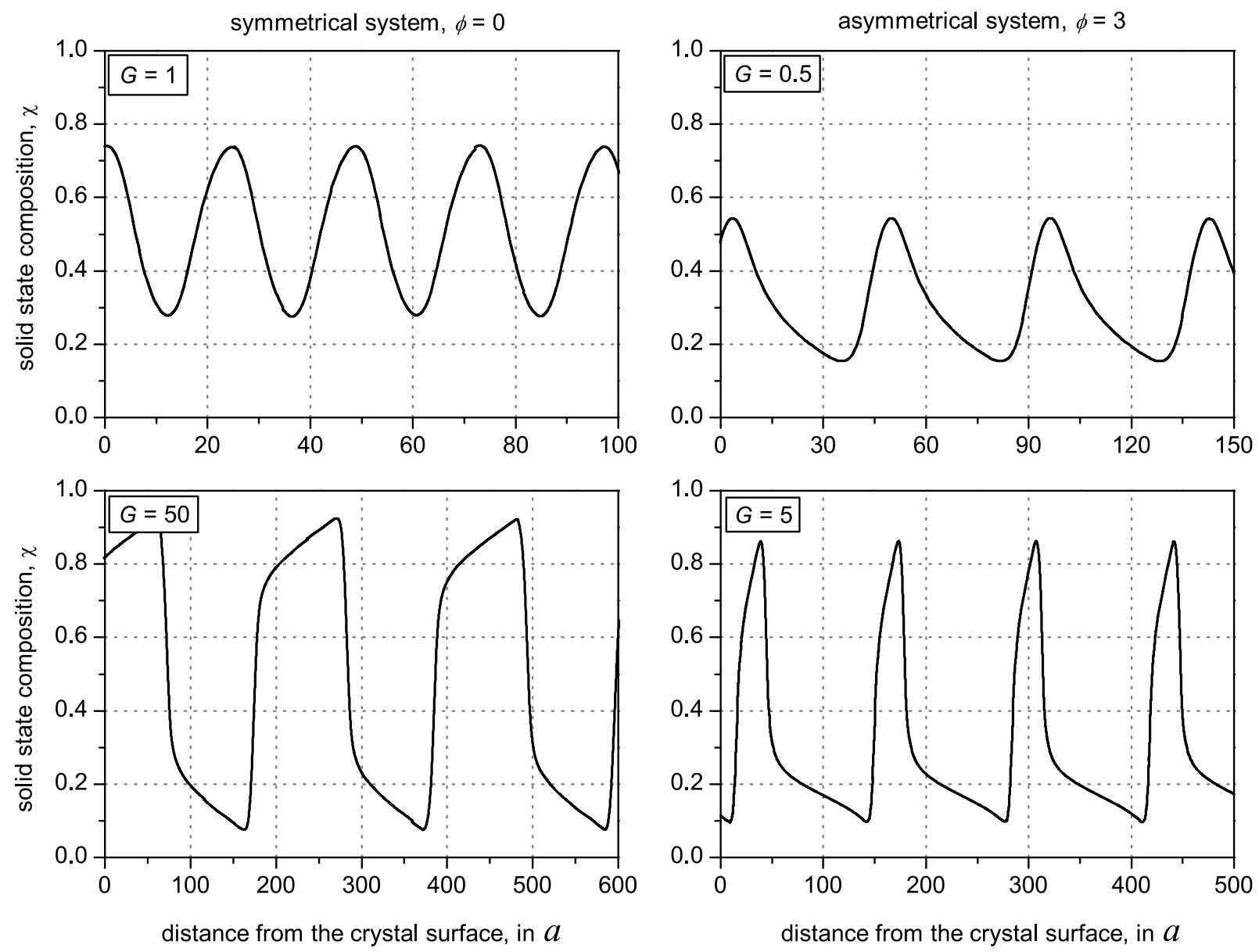

FIG. 7: Spatial patterns formed in the growing crystal.

on the crystal side is essential for the existence of OZ. In addition, we perform the stability analysis without further approximations. This may be essential because the oscillatory dynamics can stem from the counteraction of strong "forces" mutually compensating each other at the first approximation. We would like to mention that in [16] the system of equations has been numerically solved without invoking the boundary layer approximation. On a qualitative level similar results were obtained as compared to the analytical treatment within the boundary layer approximation. (v) The surface roughness parameter, used in [15], is not necessary in the present analysis.

The proposed growth mechanism is valid for $a \ll l_{s} \ll$ $l$. In the limit of $l_{s} \approx a$ incorporation would be governed by the local crystal composition, again. In case of $l_{s} \approx l$ desorption can be neglected and nearly every adatom will be incorporated regardless of its type and $\chi$. This would result in crystals exhibiting the stoichiometric compositions 28] of the influx.

The present model cannot exhibit the bistability found in 15., because the composition of the only stationary point is determined by the influx ratio. Any crystal growth with a composition different from this ratio will result in a buildup of the currently "disfavored" component until its growth rate increases. Thus the oscillations have to revolve around or run into this fixed point. This corresponds well to the model picture, where a bistability is only possible if either the supersaturation is not high enough with respect to the minor component or if complementary crystals grow in close vicinity.

The time scales of diffusion and crystal growth define the qualitative behavior of the oscillations. At very low concentrations the crystal growth rate and consequently the changes in crystal composition are very slow. The diffusive processes on the other hand are fast enough to counteract this and no oscillatory behavior is observed. With rising solute concentrations the growth rate increases as well, whereas the characteristic speed of diffusion stays constant resulting in soft transitions and sinusoidal oscillations. At even higher concentrations, the speed of crystal composition change supercedes the diffuse reaction of the solution by many orders, creating sharp transitions, closely following $N_{\chi}(\chi)$. This qualitative description coincides with the results from the linear stability analysis At low influx it is stable possessing two imaginary eigenvalues with negative real parts. At $G_{c}$ the 
real part changes sign and the system becomes unstable.

Finally, the numerical calculation of asymmetrical systems show the stabilization of a formerly unstable branch of $N_{\chi}(\chi)$. If the fixed point is shifted far enough onto the stable branch, the trajectories slowly approach it along the nullcline, but upon nearly reaching it are directed away with the onset of new oscillations. The amplitude of these oscillations can have continuously growing character or they can exhibit certain characteristic values. This might be the onset of frequency doubling and provide a route into chaotic behavior of the system. This however, is beyond the scope of the present manuscript.

\section{CONCLUSION}

The presented boundary reaction diffusion model, describing OZ of crystals grown from solution. Thereby it applies a mechanism including surface diffusion, which can be derived from microscopic properties. OZ arises due to diffusive build up of the disfavored component above the crystal until the autocatalytic growth process in combination with the large population turn the crystal composition in favor of the formerly disfavored species. A linear stability analysis was carried out without further approximations. The results correspond to the experimental findings of a supersaturation threshold before the onset of OZ. Numerical simulations of asymmetric cases show a stabilization of previously unstable parts of the system.

\section{Acknowledgments}

Felix Kalischewski would like to thank the "Fonds der Chemischen Industrie" and the "NRW Graduate School of Chemistry" for financial support. Ihor Lubashevsky appreciates the financial support of the SFB 458 and the University of Münster as well as the partial support of RFBR Grant 04-02-81059. We thank Prof. Putnis and
Prof. Purwins for helpful discussions.

\section{EIGENVALUE EQUATION FOR THE BOUNDARY-REACTION DIFFUSION MODEL}

Let us convert the system of equations (33) and (34) into a form more appropriate for its analysis. By virtue of (33) both the wave numbers $p_{i}$ have the same argument $\psi \in(-\pi / 2 ; \pi / 2)$, i.e. $p_{i}=\left|p_{i}\right| \exp (i \psi)$ whose twice value gives the argument of $\gamma$ and their absolute values obey the equality $\left|p_{i}\right|=\left(|\gamma| / D_{i}\right)^{1 / 2}$.

A new variable $\zeta>0$ and parameter $\Delta$ introduced as follows

$$
\begin{aligned}
\zeta^{2} & =\frac{\sqrt{D_{1} D_{2}} \tau_{1} \tau_{2}}{a^{2}}|\gamma|=\frac{\sqrt{D_{1} D_{2}} \tau^{2}}{a^{2}} e^{\phi(1-2 \chi)+\theta}|\gamma| \\
\Delta^{2} & =\frac{\sqrt{D_{1}} \tau_{1}}{\sqrt{D_{2}} \tau_{2}}=\sqrt{\frac{D_{1}}{D_{2}}} f_{\eta}^{-2} e^{-\phi+\theta(1-2 \chi)}
\end{aligned}
$$

enable us, first, to write

$$
D_{1} \tau_{1}\left|p_{1}\right|=a \zeta \Delta, \quad D_{2} \tau_{2}\left|p_{2}\right|=a \zeta / \Delta
$$

and, then, to represent equation (34) in the form

$$
\begin{aligned}
& \frac{\zeta^{2}}{g} e^{\mathrm{i} 2 \psi}=-1+\chi(1-\chi) \\
& \times\left[(\theta+\phi) \frac{(\zeta \Delta) e^{\mathrm{i} \psi}}{(\zeta \Delta) e^{\mathrm{i} \psi}+1}+(\theta-\phi) \frac{(\zeta / \Delta) e^{\mathrm{i} \psi}}{(\zeta / \Delta) e^{\mathrm{i} \psi}+1}\right],
\end{aligned}
$$

where the parameter $g$ stands for the dimensionless species flux casing the crystal growth

$$
g=\sqrt{D_{1} D_{2}} \tau_{1} \tau_{2} G=\sqrt{D_{1} D_{2}} \tau^{2} e^{\phi(1-2 \chi)+\theta} G .
$$

Finally the split of equality (53) into the real and imaginary parts yields the desired coupled equations specifying actually the eigenvalue $\gamma$

$$
\begin{aligned}
& \frac{\zeta^{2}}{g} \cos (2 \psi)=-1+\chi(1-\chi)\left[(\theta+\phi) \frac{(\zeta \Delta)(\zeta \Delta+\cos \psi)}{(\zeta \Delta+\cos \psi)^{2}+\sin ^{2} \psi}+(\theta-\phi) \frac{(\zeta / \Delta)(\zeta / \Delta+\cos \psi)}{(\zeta / \Delta+\cos \psi)^{2}+\sin ^{2} \psi}\right] \\
& \frac{\zeta^{2}}{g} \sin (2 \psi)=\chi(1-\chi) \sin \psi\left[(\theta+\phi) \frac{(\zeta \Delta)}{(\zeta \Delta+\cos \psi)^{2}+\sin ^{2} \psi}+(\theta-\phi) \frac{(\zeta / \Delta)}{(\zeta / \Delta+\cos \psi)^{2}+\sin ^{2} \psi}\right]
\end{aligned}
$$

As follows from equations (55) the value $\zeta=0$ is not a root of this system. So the considered instability is to arise through the real part of $\gamma$ changing the sign with its imaginary part having some finite value. Therefore to find the instability boundary $\operatorname{Re} \gamma=0$ we can set $\psi=\pi / 4$ in the given equations. In this way the former equation (55a) converts into one specifying the value of $\zeta$ for chosen values of the parameters $\chi, \theta, \phi$, and $\Delta$ 


$$
(\theta+\phi) \frac{(\sqrt{2} \zeta \Delta)(\sqrt{2} \zeta \Delta+1)}{(\sqrt{2} \zeta \Delta+1)^{2}+1}+(\theta-\phi) \frac{(\sqrt{2} \zeta / \Delta)(\sqrt{2} \zeta / \Delta+1)}{(\sqrt{2} \zeta / \Delta+1)^{2}+1}=\frac{1}{\chi(1-\chi)},
$$

whereas the latter equation determines the critical value of the diffusion flux $g_{c}$

$$
g_{c}=\frac{\zeta}{\sqrt{2} \chi(1-\chi)}\left[(\theta+\phi) \frac{\Delta}{(\sqrt{2} \zeta \Delta+1)^{2}+1}+(\theta-\phi) \frac{1 / \Delta}{(\sqrt{2} \zeta / \Delta+1)^{2}+1}\right]^{-1} .
$$

Returning to the dimensional variables we get expressions (36) and (37).

[1] M. Shore and A. D. Fowler, Can. Mineral. 34, 1111 (1996).

[2] R. J. Reeder, R. O. Fagioli, and W. J. Meyers, EarthScience Reviews 29, 39 (1990).

[3] A. Putnis, L. Fernandez-Diaz, and M. Prieto, Nature 358, 743 (1992).

[4] C. M. Pina, M. Enders, and A. Putnis, Chemical Geology 168, 195 (2000).

[5] M. Prieto, A. Putnis, and L. Fernandez-Diaz, Geological Magazine 130, 289 (1993).

[6] A. Putnis, Private Communications .

[7] P. J. Ortoleva, Geochemical Self-Organization (Oxford University Press, New York, 1994).

[8] P. J. Ortoleva, Earth-Science Reviews 29, 3 (1990).

[9] C. S. Haase, J. Chadam, D. Feinn, and P. Ortoleva, Science 209, 272 (1980).

[10] Y. Wang and E. Merino, Geochimica et Chosmochimica Acta 54, 1627 (1990).

[11] Y. Wang and E. Merino, Geochimica et Cosmochimica Acta 56, 587 (1992).

[12] Y. Wang and E. Merino, Journal of Petrology 34, 369 (1993).

[13] I. L'Heureux, American Mineralogist 79, 885 (1994).

[14] J.-H. Wang and J.-P. Wu, Eur. J. Mineral. 7, 1089 (1995).

[15] I. L'Heureux and B. Jamtveit, Geochimica et Comsochimica Acta 66, 417 (2002).

[16] S. Katsev and I. L'Heureux, Physical Review E 66
(2002).

[17] I. L'Heureux and S. Katsev, Chemical Geology 225, 230 (2006).

[18] I. V. Markov, Crystal Growth for Beginners (World Scientific, 1998).

[19] W. K. Burton, N. Cabrera, and F. C. Frank, Philosophical Transactions of the Royal Society of London 243, 299 (1951).

[20] C. M. Pina, U. Becker, P. Risthaus, D. Bosbach, and A. Putnis, Nature 395, 483 (1998).

[21] F. Kalischewski, Diploma Thesis (2004).

[22] G. H. Gilmer, R. Ghez, and N. Cabrera, Journal of Crystal Growth 8, 79 (1971).

[23] A. Karma and A. Sarkissian, Physical Review E 47, 512 (1993).

[24] V. V. Gafiychuk, I. A. Lubashevsky, and V. V. Osoipov, Sov. Phys. Crystallogr. 37, 447 (1992).

[25] I. A. Lubashevsky, V. V. Gafiychuk, and M. G. Keijan, arXiv:adap-org/9810002 (1998)

[26] V. Gafiychuk and I. Lubashevsky, Nonlinear Theory and Modeling of the Free Boundary Problems Arising in Distributed Media (VNTL Publishers, Lviv, 2001).

[27] E. Izhikevich, SIAM J. Appl. Math. 60, 1789 (2000).

[28] M. Prieto, A. Fernandez-Gonzalez, A. Putnis, and L. Fernandez-Diaz, Geochimica et Cosmochimica Acta 61, 3383 (1997). 